TRANSACTIONS OF THE

AMERICAN MATHEMATICAL SOCIETY

Volume 349, Number 2, February 1997, Pages 657-673

S 0002-9947(97)01857-6

\title{
TANGENTIAL FLATNESS AND GLOBAL RIGIDITY OF HIGHER RANK LATTICE ACTIONS
}

\author{
NANTIAN QIAN
}

\begin{abstract}
We establish the continuous tangential flatness for orientable weakly Cartan actions of higher rank lattices. As a corollary, we obtain the global rigidity of Anosov Cartan actions.
\end{abstract}

\section{INTRODUCTION AND THE STATEMENT OF RESULTS}

Throughout this paper we assume that $G$ is a connected semisimple Lie group with finite center and without compact factors, each simple factor of which has real rank at least 2; assume that $\Gamma$ is a lattice in $G$, i.e., a discrete subgroup such that the quotient $G / \Gamma$ has finite Haar measure. We also assume that $M$ is an $n$-dimensional smooth compact manifold and $\rho$ is a $C^{r}$-action of $\Gamma$ on $M$ (or equivalently, $\rho$ is a homomorphism from $\Gamma$ to $\left.\operatorname{Diff}^{r}(M)\right)$ for an integer $r \geq 1$.

In this paper, we investigate the global rigidity of $\Gamma$ actions on $M$. We recall that in his 1986 address in ICM [Z3], Zimmer asked whether all ergodic, volumepreserving actions $\rho$ of higher rank lattices can be built up from the actions of three types by simple algebraic constructions such as products, suspensions, finite extensions or compact quotients (it is referred to later in this paper as the standard list): (1) Isometric actions (i.e., $\rho(\Gamma)$ has compact closure in $\left.\operatorname{Diff}^{r}(M)\right)$; (2) Affine actions on compact nilmanifolds; (3) Left translations on compact quotients $H / \Lambda$ via homomorphism $\Gamma \rightarrow H$, where $H$ is a connected Lie group, $\Lambda \subset H$ is a cocompact lattice. A new class of actions discovered by Katok and Lewis [KL2], which are essentially obtained by introducing lower-dimensional non-affine behavior using a sophisticated approach to "blow-up" periodic points, suggested one can only hope the above examples to be the building-blocks on an open dense set. Katok and Lewis, Zimmer conjectured that there is a smooth invariant connection on an open dense set [KL2] [Z5]. We first make precise the notion of global rigidity in this paper. (We remark that this notion applies to examples in (2) and (3) in the standard list. For other situations we need a more general definition.)

Let $L$ be a connected, simply connected Lie group with Lie algebra $\mathcal{L}$ and $\Lambda$ is a cocompact lattice of $L$. We denote by $\operatorname{Aff}(L)$ the set of all $C^{1}$-diffeomorphisms sending right-invariant vector fields into right-invariant vector fields on $L$, and $\operatorname{Aff}(L / \Lambda)$ the set of all $C^{1}$-diffeomorphisms of $L / \Lambda$ that lift to diffeomorphisms in $\operatorname{Aff}(L)$ of $L$. It is easy to see that a diffeomorphism $F \in \operatorname{Aff}(L)$ iff $F=A a$ where $A$ is an automorphism of $L$ and $a$ is a left translation on $L$; hence $F \in \operatorname{Diff}^{\infty}(L)$. As

Received by the editors December 13, 1994.

1991 Mathematics Subject Classification. Primary 22E40, 58E40.

Key words and phrases. Rigidity of group actions, Lie groups, dynamical systems.

(c)1997 American Mathematical Society 
observed by $[\mathrm{F}]$, there exists a naturally defined connection $\nabla$ on $L / \Lambda$ - by the requirement that the right invariant vector fields are parallel - such that $\operatorname{Aff}(L / \Lambda)$ coincides with the group of all $\nabla$-preserving diffeomorphisms.

Let $\rho$ be a smooth action of $\Gamma$ on $M$. We say that the action $\rho$ is $C^{r}$-globally rigid if there exist

(GR1) a finite covering $\tilde{M}$ of $M$,

(GR2) a subgroup $\Gamma_{0} \subset \Gamma$ of finite index,

(GR3) a connected, simply connected Lie group $L$ and a cocompact lattice $\Lambda$ in $L$,

(GR4) a homomorphism $\rho_{0}: \Gamma_{0} \rightarrow \operatorname{Aff}(L / \Lambda)$,

(GR5) a $C^{r}$-diffeomorphism $\phi: \tilde{M} \rightarrow L / \Lambda$,

such that the lift of the action $\rho$ of $\Gamma_{0}$ to that on $\tilde{M}$ (still denoted by $\rho$ ) satisfies $\rho(\gamma)=\phi^{-1} \rho_{0}(\gamma) \phi$ for all $\gamma \in \Gamma_{0}$.

The first global rigidity result is established by Katok, Lewis and Zimmer [KLZ]. They show, among other things, that an Anosov volume-preserving $S L(n, \mathbb{Z})$ action on $\mathbb{T}^{n}$ is $C^{\infty}$-globally rigid. Their proof uses Zimmer's cocycle superrigidity to obtain measure-theoretic information of the action and then uses the theory of hyperbolic dynamical systems to obtain the continuous and the smooth information. To use their argument to establish the global rigidity for action $\rho$, therefore, the presence of the hyperbolicity of the action is essential. Also to overcome the technical difficulties, additional assumptions about the action are needed. We thus consider a class of actions called weakly Cartan actions.

We say that an action $\rho$ of $\Gamma$ on $M$ is (orientable) weakly Cartan if there exist

(WC1) a continuous tangent bundle splitting $T M=E_{1} \oplus \cdots \oplus E_{n}$,

(WC2) $n$ elements $\gamma_{1}, \ldots, \gamma_{n}$, such that for each $i=1, \ldots, n, \rho\left(\gamma_{i}\right)$ is partially hyperbolic,

(WC3) $E_{i}$ is the 1-dimensional strongest (orientable) distribution for $\rho\left(\gamma_{i}\right)$.

One common feature of the examples (2) and (3) in the standard list is the $C^{\infty}$ tangential flatness. A smooth action is said to be $C^{r}$-tangential flat if there exists a $C^{r}$-framing $\sigma(x)$ for the tangent bundle such that the induced tangent action is given by a homomorphism $\pi: \Gamma \rightarrow G L(n, \mathbb{R})$; or equivalently, with respect to the framing the induced action on the fibers of $T M$ is independent of the base point $x \in M$.

We will establish the following continuous tangential flatness result in $\S 2$.

Theorem 1.1. Let $\Gamma \subset G$ be a higher rank lattice, where each simple factor of $G$ has real rank at least 2 . Let $\rho$ be an ergodic $C^{1+\alpha}$-action of $\Gamma$ on $M$ with respect to a probability measure $m$ equivalent to a Lebesgue measure. Assume that $\rho$ is orientable weakly Cartan. Then there exists a subgroup $\Gamma_{0} \subset \Gamma$ of finite index such that $\left.\rho\right|_{\Gamma_{0}}$ is $C^{\alpha}$-tangentially flat.

The importance of the tangential flatness of actions is an observation from a result of Feres $[\mathrm{F}]$ and an implication of the ergodicity of the group action. Feres observed that if there exist $n(n=\operatorname{dim}(M))$ non-vanishing $C^{r}$-vector fields on $M$ that are generators of an $n$-dimensional Lie algebra $\mathcal{L}$, and if moreover they form a linearizing framing for action $\rho$, then $\rho$ is $C^{r+1}$-globally rigid, for $r \geq 1$ if $\mathcal{L}$ is abelian and for $r \geq 2$ otherwise. 
We show for an ergodic tangentially flat action that the $C^{r}$-smoothness $(r \geq 1)$ of the linearizing framing is enough for the global rigidity. The following result is proved in $\S 3$.

Theorem 1.2. Let $\Gamma, G$ be as in Theorem 1.1, $r \geq 1$ be an integer, $\rho$ be an ergodic tangentially flat $C^{r+1}$-action of $\Gamma$ on $M$ associated with $\pi: \Gamma \rightarrow G L(n, \mathbb{R})$ with respect to a $C^{r}$ framing $\left\{X_{1}, \ldots, X_{n}\right\}$ (necessarily preserves a $C^{r}$ volume form). Assume that $\pi$ extends to a continuous homomorphism $G \rightarrow G L(n, \mathbb{R})$. Then $\rho$ is $C^{r+1}$-globally rigid; i.e., there exist a connected, simply connected Lie group $L$ and a cocompact lattice $\Lambda$ in $L$, a homomorphism $\rho_{0}: \Gamma \rightarrow A f f(L / \Lambda)$, a $C^{r+1}$ _ diffeomorphism $\phi: M \rightarrow L / \Lambda$, such that $\rho(\gamma)=\phi^{-1} \rho_{0}(\gamma) \phi$.

Global rigidity of Cartan action (in the sense of Hurder [Hu1], [Hu2]) and other corollaries will be discussed in $\S 4$.

The author would like to thank G.A. Margulis, G.D. Mostow, C.-B. Yue and R.J. Zimmer for helpful discussions.

\section{2. $C^{0}$-TANGENTiAL FLATNESS}

Let $M$ be a smooth compact manifold. We choose a measurable framing in the following way. If there exists an orientable weakly Cartan action on $M$, we choose one continuous vector field from each of the one-dimensional distributions defining orientable weakly Cartan structure. Otherwise if there exists a tangentially flat action on $M$, we let the framing be the continuous framing which gives the tangentially flat structure. In other cases we let the framing be a measurable framing smooth in an open dense subset of $M$. In this section, if no mention is made of a framing in a statement, we always assume that the framing is the framing we choose above, and denote it by $\sigma_{0}(x)$.

In this section we always assume that

(A1) $G$ and $\Gamma$ are as in the beginning of this paper;

(A2) $\tilde{G}$ is the universal covering Lie group of $G, \tilde{\mathbf{G}}$ the maximal algebraic factor of $\tilde{G}$ (or more precisely, let $G$ modulo the center be the group of $\mathbb{R}$-points of an algebraic group $\mathbf{G}^{\prime}$; then the algebraic universal cover of $\mathbf{G}^{\prime}$ is $\tilde{\mathbf{G}}$ );

(A3) $M$ is an $n$-dimensional compact smooth manifold, and $m$ is a probability measure on $M$ taking positive values for open sets;

(A4) if $\pi$ is a continuous homomorphism of $G$ to $G L(n, \mathbb{R})$ factoring through the center, then $\tilde{\pi}$ is the lift of $\pi$ to a homomorphism $\tilde{G} \rightarrow G L(n, \mathbb{R})$.

We state a corollary of Zimmer's cocycle superrigidity.

Lemma 2.1. Let $G$ and $\Gamma$ be as in (A1), $M$ and $m$ be as in (A3). Let $\rho$ be a $C^{1}$ ergodic action of $\Gamma$ on $M$ preserving a probability measure $m$ and $\alpha$ be the derivative cocycle. Then there exist

1. an algebraic $\mathbb{R}$-group $\mathbf{H}$ such that the algebraic hull of $\alpha$ is $\mathbf{H}_{\mathbb{R}}$;

2. a cocycle $\beta$ over $\rho$ taking values in $\mathbf{H}_{\mathbb{R}}$ such that $\alpha$ is equivalent to $\beta$.

If we define a twisted action $\rho^{\prime}$ on $M \times \mathbf{H}_{\mathbb{R}} /\left(\mathbf{H}^{0}\right)_{\mathbb{R}}$ by $(x,[h], \gamma) \mapsto(\rho(\gamma) x, \beta(x, \gamma)[h])$, define a cocycle $\beta^{\prime}$ over $\rho^{\prime}$ by $((x,[h]), \gamma) \mapsto \beta(x, \gamma)$ and define a cocycle $\alpha^{\prime}$ over $\rho^{\prime}$ by $(x,[h], \gamma) \mapsto \alpha(x, \gamma)$, then

3. $\rho^{\prime}$ is ergodic with respect to $m \times \mu$ where $\mu$ is the normalized counting measure on $\mathbf{H}_{\mathbb{R}} /\left(\mathbf{H}^{0}\right)_{\mathbb{R}}$ (recall that $\mathbf{H}_{\mathbb{R}} /\left(\mathbf{H}^{0}\right)_{\mathbb{R}}$ is necessarily finite);

4. the algebraic hull of $\beta^{\prime}$ is $\left(\mathbf{H}^{0}\right)_{\mathbb{R}}$; 
5. let $\tilde{G}$ be as in (A2). Then $\beta^{\prime}$ is equivalent to the cocycle defined by $\delta$ : $(x,[h], \gamma) \mapsto \tilde{\pi}(\tilde{\gamma}) b(x,[h], \tilde{\gamma})$, where $\tilde{\pi}$ is a homomorphism from $\tilde{G}$ to $\left(\mathbf{H}^{0}\right)_{\mathbb{R}}($ which factors to a rational homomorphism of the maximal algebraic factor of $\tilde{G}$ ) and $b(x,[h], \tilde{g})$ is a measurable map taking values in a compact normal subgroup of $\left(\mathbf{H}^{0}\right)_{\mathbb{R}}(\tilde{\gamma}$ projects to $\gamma$ under the covering map) and moreover, $b(x,[h], \tilde{\gamma})$ and $\tilde{\pi}(\tilde{\gamma})$ commute for a.e $(x,[h]) \in M \times \mathbf{H}_{\mathbb{R}} /\left(\mathbf{H}^{0}\right)_{\mathbb{R}}$ and all $\gamma \in \Gamma$;

6. $\alpha^{\prime}$ is equivalent to $\delta$.

Proof. The results follow from Zimmer's cocycle superrigidity (Theorem 2.2 of [Z1]) and standard argument by Zimmer, Lewis and others. In fact more general results are true (Lemma 3.1 of [QZ]). We prove a special case for the convenience of the reader: we assume that $\Gamma \subset G=\mathbf{G}_{\mathbb{R}}^{0}$ where $\mathbf{G}_{\mathbb{R}}^{0}$ is the (Hausdorff) connected component of $\mathbb{R}$-points of a connected almost $\mathbb{R}$-simple $\mathbb{R}$-group $\mathbf{G}$ with $\mathbb{R}$-rank $(G) \geq 2$ and uses a weaker form of Zimmer's cocycle superrigidity (Theorem 9.4.14 of [Z2]).

Let $\alpha: M \times G \rightarrow G L(n, \mathbb{R}) \subset G L(n, \mathbb{C})$ be the derivative cocycle and $\mathbf{H}_{\mathbb{R}}$ be the algebraic hull of $\alpha$. Then $\mathbf{H}$ is reductive and the real points of the center $(Z(\mathbf{H}))_{\mathbb{R}}$ of $\mathbf{H}$ are compact (by Zimmer [Z4] for cocompact $\Gamma$, and Lewis [L] for non-cocompact $\Gamma)$. Let $S=M \times \mathbf{H}_{\mathbb{R}} /\left(\mathbf{H}^{0}\right)_{\mathbb{R}}$. Then the algebraic hull of $\beta^{\prime}$ is $\left(\mathbf{H}^{0}\right)_{\mathbb{R}}$ (9.2.6 in [Z2]). Let $L=\mathbf{H}^{0}$; we can write the $\mathbb{R}$-group $\mathbf{L} / Z(\mathbf{L})$ as a product of semisimple $\mathbb{R}$-groups $\mathbf{L} / Z(\mathbf{L})=\mathbf{L}_{1} \times \mathbf{L}_{2}$ such that $\left(\mathbf{L}_{2}\right)_{\mathbb{R}}$ is compact, and $\left(\mathbf{L}_{1}\right)_{\mathbb{R}}$ is center free and with no compact factors. Let $q_{1}: \mathbf{L}_{\mathbb{R}} \rightarrow\left(\mathbf{L}_{1}\right)_{\mathbb{R}}$ be the projection. Then $q_{1} \circ \beta^{\prime}: S \times \Gamma \rightarrow\left(\mathbf{L}_{1}\right)_{\mathbb{R}}$ is a cocycle with algebraic hull $\left(\mathbf{L}_{1}\right)_{\mathbb{R}}$, and by superrigidity theorem for cocycles (9.4.14 of [Z2]), there is a rational homomorphism $\pi: \mathbf{G} \rightarrow \mathbf{L}_{1}$ defined over $\mathbb{R}$ such that $q_{1} \circ \beta^{\prime} \cong \alpha_{\pi \mid \Gamma}$ where $\alpha_{\pi}$ is the cocycle $\alpha_{\pi}(s, g)=\pi(g)$. Thus we can write for $\gamma \in \Gamma$ and a.e. $s \in S, \phi(s) q_{1}\left(\beta^{\prime}(s, \gamma)\right) \phi(s \gamma)^{-1}=\pi(\gamma)$ where $\phi: S \rightarrow\left(\mathbf{L}_{1}\right)_{\mathbb{R}}$ is Borel. We can choose a Borel section $f:\left(\mathbf{L}_{1}\right)_{\mathbb{R}} \rightarrow \mathbf{L}_{\mathbb{R}}$ of $q_{1}$. Then $q_{1}\left(f(\phi(s)) \beta^{\prime}(s, \gamma) f(\phi(s \gamma))^{-1}\right)=\pi(\gamma)$. In other words, replacing $\beta^{\prime}$ by an equivalent cocycle $\delta: S \times G \rightarrow \mathbf{L}_{\mathbb{R}}$, we have $q_{1}(\delta(s, \gamma))=\pi(\gamma)$.

We can consider $\pi$ as a homomorphism $\pi: \tilde{\mathbf{G}} \rightarrow \mathbf{L}_{1}(\subset \mathbf{L} / Z(\mathbf{L}))$, where $\tilde{\mathbf{G}}$ is the algebraic universal covering group of $G$. Since $[\mathbf{L}, \mathbf{L}] \cap Z(\mathbf{L})$ is finite, we can lift $\pi$ to a homomorphism $\tilde{\pi}: \tilde{\mathbf{G}} \rightarrow[\mathbf{L}, \mathbf{L}] \subset G L(n, \mathbb{C})$ defined over $\mathbb{R}$. Thus for each $\gamma \in \Gamma$, we have $q_{1}(\delta(s, \gamma))=q_{1}(\tilde{\pi}(\tilde{\gamma}))$ for a.e $s$, where $\tilde{\gamma} \in \tilde{\mathbf{G}}_{\mathbb{R}}$ projects to $\gamma$ under the covering map. However, since $\left(\mathbf{L}_{2}\right)_{\mathbb{R}}$ and $Z(\mathbf{L})_{\mathbb{R}}$ are compact, $\left(\text { ker } q_{1}\right)_{\mathbb{R}}$ is compact. Thus, $\tilde{\pi}(\tilde{\gamma})=\delta(s, \gamma) b^{\prime}(s, \tilde{\gamma})$ and therefore $\delta(s, \gamma)=\tilde{\pi}(\tilde{\gamma}) b(s, \tilde{\gamma})$ where $b$ is a measurable function taking values in a compact normal subgroup $\left(\text { ker } q_{1}\right)_{\mathbb{R}}$ of $\mathbf{L}_{\mathbb{R}}$.

We now show that $\left(\operatorname{ker} q_{1}\right)_{\mathbb{R}}$ and $\tilde{\pi}\left(\tilde{\mathbf{G}}_{\mathbb{R}}\right)$ centralize each other. We first note that $\mathbf{L}=\mathbf{H}^{0}$ is reductive $\mathbb{R}$-group. Therefore, $\mathbf{H}^{0}$ can be decomposed into an almost direct product of almost $\mathbb{R}$-simple factors and the center $Z\left(\mathbf{H}^{0}\right)$; i.e., $\mathbf{H}^{0}=$ $\mathbf{H}_{1} \ldots \mathbf{H}_{i_{1}} \mathbf{H}_{i_{1}+1} \cdot \ldots \mathbf{H}_{i_{2}} \cdot Z\left(\mathbf{H}^{0}\right)$ where for each $j, \mathbf{H}_{j}$ is an almost $\mathbb{R}$-simple $\mathbb{R}$-group, for $j=1, \ldots, i_{1}\left(\mathbf{H}_{j}\right)_{\mathbb{R}}$ is non-compact, and for $j=i_{1}+1, \ldots, i_{2}\left(\mathbf{H}_{j}\right)_{\mathbb{R}}$ is compact, and the intersection of any two distinct factors $\mathbf{H}_{i} \cap \mathbf{H}_{j}$ is a finite subgroup of the center $Z\left(\mathbf{H}^{0}\right)$ and hence they centralize each other. Since ker $q_{1}$ is a normal subgroup defined over $\mathbb{R}$ and $\left(\text { ker } q_{1}\right)_{\mathbb{R}}$ is compact, ker $q_{1}$ is the product of some $\mathbf{H}_{j}$ 's with $j=i_{1}+1, \ldots, i_{2}$ and/or $Z\left(\mathbf{H}^{0}\right)$. Since the $\mathbb{R}$-rank of each of the simple factors of $\tilde{\mathbf{G}}_{\mathbb{R}} \geq 2$, there is no non-trivial rational homomorphism from $\tilde{\mathbf{G}}$ to $\mathbf{H}_{j}$ (for $j=i_{1}+1, \ldots, i_{2}$ ) or $Z\left(\mathbf{H}^{0}\right)$ (except for a possible homomorphism from $\tilde{\mathbf{G}}$ to $Z\left(\mathbf{H}^{0}\right)$ with a finite image). Thus the image of the projection from $\tilde{\pi}(\tilde{\mathbf{G}})$ to 
$\mathbf{H}_{i_{1}+1} \cdot \ldots \mathbf{H}_{i_{2}} \cdot Z\left(\mathbf{H}^{0}\right)$ is a subgroup in $Z\left(\mathbf{H}^{0}\right)$. Therefore, the image of $b$ and the image of $\tilde{\pi}$ centralize each other.

Lemma 2.2. Let $G$ and $\Gamma$ be as in (A1), $M$ and $m$ be as in (A3). Let $\mathbf{H}$ be an algebraic $\mathbb{R}$-group such that the algebraic hull of the derivative cocycle is $\mathbf{H}_{\mathbb{R}}$. Let $\rho$ be an ergodic, orientable weakly Cartan action of $\Gamma$ on $M$ preserving $m$. Let $\beta^{\prime}$ be the cocycle over the twisted action $\rho^{\prime}$ as in Lemma 2.1. Then $\beta^{\prime}$ is equivalent to a cocycle $\delta$ over $\rho^{\prime}$ defined by $(x,[h], \gamma) \mapsto \tilde{\pi}(\tilde{\gamma}) b(x,[h], \tilde{\gamma})$ where $b(x,[h], \tilde{\gamma})$ takes finitely many values in $Z\left(\mathbf{H}^{0}\right)_{\mathbb{R}}$.

Proof. Let $\mathbf{H}, \beta, \tilde{\pi}, b$ be as in Lemma 2.1. By passing to a finite cover of $M$ we may assume that $\mathbf{H}$ is Zariski connected. From Lemma 2.1 we know that $\beta^{\prime}$ is equivalent to a cocycle $\delta:(x,[h], \gamma) \mapsto \tilde{\pi}(\tilde{\gamma}) b(x,[h], \tilde{\gamma})$, and the image of $b$ centralizes the image of $\tilde{\pi}$.

We now show that each irreducible constituent of $\tilde{\pi}:\left(\tilde{\mathbf{G}}_{\mathbb{R}}\right)^{0} \rightarrow G L(n, \mathbb{R})$ is multiplicity free. Let $\tilde{\pi}=l_{1} \tilde{\pi}_{1} \oplus \cdots \oplus l_{k} \tilde{\pi}_{k}$ be the irreducible decomposition, and for each $i=1, \ldots, k$ let $V_{i} \subset \mathbb{R}^{n}$ be the invariant space corresponding to $l_{1} \tilde{\pi}_{i}$. For a typical $(x,[h])$ let $b_{0}=b(x,[h], \tilde{\gamma})$. Since $b_{0}$ commutes with $\tilde{\pi}(\tilde{\gamma}), b_{0} V_{i}=V_{i}$. Hence $V_{i}$ is both $\tilde{\pi}$-invariant and $b$-invariant. We interpret above in terms of the measurable invariant subbundle in $T\left(M \times \mathbf{H}_{\mathbb{R}} /\left(\mathbf{H}^{0}\right)_{\mathbb{R}}\right.$ as follows. Let $\alpha^{\prime}$ be the lift of derivative cocycle $\alpha$. There exist $k$ measurable $\alpha^{\prime}$-invariant subbundles $F_{1}, \ldots, F_{k}$ of $T\left(M \times \mathbf{H}_{\mathbb{R}} /\left(\mathbf{H}^{0}\right)_{\mathbb{R}}\right)$, and for each $i=1, \ldots, k$ a measurable framing for $F_{i}$, such that with respect to the framing $\left.\alpha^{\prime}\right|_{F_{i}}(x,[h], \gamma)=l_{i} \tilde{\pi}_{i}(\tilde{\gamma}) b(x,[h], \tilde{\gamma})$.

Since $\rho$ is orientable weakly Cartan, there exist a continuous tangent bundle splitting $T M=E_{1} \oplus \cdots \oplus E_{n}, n$ elements $\gamma_{1}, \ldots, \gamma_{n}$, such that for each $i=1, \ldots, n$, $\rho\left(\gamma_{i}\right)$ is partially hyperbolic, and $E_{i}$ is the 1-dimensional strongest (orientable) distribution for $\rho_{i}$. So if $\chi_{i}(x)$ is the maximal Lyapunov exponent of $\rho\left(\gamma_{i}\right)$, then the corresponding Oceledec measurable $\rho\left(\gamma_{i}\right)$-invariant bundle is exactly one dimensional continuous distribution $E_{i}$. We lift naturally $\chi_{i}(x), E_{i}(x)$ to $M \times \mathbf{H}_{\mathbb{R}} /\left(\mathbf{H}^{0}\right)_{\mathbb{R}}$, let $\chi_{i}(x,[h])=\chi_{i}(x)$ and $E_{i}(x,[h])=E_{i}(x)$. It follows from Lemma 2.4 of [KLZ] that the maximal Lyapunov exponent $\chi_{i}^{\prime}$ of $\rho\left(\gamma_{i}\right)$ is the maximum of the absolute values of $\tilde{\pi}(\tilde{\gamma})$, and its Oceledec measurable $\rho\left(\gamma_{i}\right)$-invariant bundle $E_{i}^{\prime}$ corresponds to (generalized) eigenspace of $\chi_{i}^{\prime}$. Hence $E_{i}^{\prime}(x,[h])=E_{i}(x)$ and $\chi_{i}^{\prime}=\chi_{i}(x)$ for almost all $(x,[h]) \in M \times \mathbf{H}_{\mathbb{R}} /\left(\mathbf{H}^{0}\right)_{\mathbb{R}}$. We thus obtain that $E_{i}$ is contained in $F_{j}$ for some $j=1, \ldots, k$; and moreover, for such $j, F_{j}$ is multiplicity free. It is easy to see that all $F_{i}$ are irreducible, since $T M=E_{1} \oplus \cdots \oplus E_{n}$.

Since each irreducible constituent of $\tilde{\pi}$ is multiplicity free, and $\tilde{\pi}(\tilde{\gamma})$ commutes with $b(x,[h], \tilde{\gamma})$, it is easy to see by Schur's lemma that $\left.b(x,[h], \tilde{\gamma})\right|_{V_{i}}$ is a scalar. Since $b$ takes values in a compact group, we have $\left.b(x,[h], \tilde{\gamma})\right|_{V_{i}} \in\left\{ \pm \operatorname{Id}_{V_{i}}\right\}$. So $b(x,[h], \tilde{\gamma}) \in \bigoplus_{i=1}^{k}\left\{ \pm \operatorname{Id}_{V_{i}}\right\}$.

We interpret Lemma 2.2 as follows: There exists a measurable framing $\sigma(x,[h])=$ $\left\{Y_{1}, \ldots, Y_{n}\right\}\left(Y_{1}, \ldots, Y_{n}\right.$ are measurable vector fields, non-vanishing and linearly independent on a conull set) on tangent bundle $T\left(M \times \mathbf{H}_{\mathbb{R}} / \mathbf{H}_{\mathbb{R}}^{0}\right)$, such that with respect to this framing the lift $\alpha^{\prime}$ of the derivative cocycle $\alpha$ (considered as a cocycle over the twisted action $\rho^{\prime}$ of $\Gamma$ on $\left.M \times \mathbf{H}_{\mathbb{R}} / \mathbf{H}_{\mathbb{R}}^{0}\right)$ is $((x,[h]), \gamma) \mapsto \tilde{\pi}(\tilde{\gamma}) b(x,[h], \gamma)$ for some $b(x,[h], \gamma)$ in a finite group $Z=\bigoplus_{i=1}^{k}\left\{ \pm \operatorname{Id}_{V_{i}}\right\}$. Since we have a standard framing $\sigma_{0}(x)$ on $T M$ and hence lift to a standard framing (again denoted by $\left.\sigma_{0}(x,[h])\right)$ on $T\left(M \times \mathbf{H}_{\mathbb{R}} /\left(\mathbf{H}^{0}\right)_{\mathbb{R}}\right)$ (from the assumption of orientable weakly Cartan), 
we may regard $\sigma(x,[h])$ as a matrix-valued function $M \times \mathbf{H}_{\mathbb{R}} /\left(\mathbf{H}^{0}\right)_{\mathbb{R}} \rightarrow G L(n, \mathbb{R})$. We denote by $\bar{\sigma}(x,[h])$ the projection of this matrix function to $\operatorname{PGL}(n, \mathbb{R})$.

Lemma 2.3. $\bar{\sigma}: M \times \mathbf{H}_{\mathbb{R}} /\left(\mathbf{H}^{0}\right)_{\mathbb{R}} \rightarrow \mathbf{P G L}(n, \mathbb{R})$ coincides almost everywhere with a continuous function independent of $[h]$.

Proof. Without loss of generality, we may assume that $\tilde{\pi}:\left(\tilde{\mathbf{G}}_{\mathbb{R}}\right)^{0} \rightarrow G L(n, \mathbb{R})$ is irreducible. For $\gamma_{1}$ we decompose $\mathbb{R}^{n}$ into characteristic subspaces for the action of $\tilde{\pi}\left(\tilde{\gamma}_{1}\right)$ :

$\mathbb{R}^{n}=\bigoplus W_{k}, \quad W_{i}=\left\{w \neq 0 \in \mathbb{R}^{n} \mid \lim _{m \rightarrow \pm \infty}(1 / m) \ln \left\|\tilde{\pi}\left(\tilde{\gamma}_{1}\right)^{m} w\right\| /\|w\| \rightarrow \chi_{k}\right\} \cup\{0\} ;$

$\chi_{k} \in \mathbb{R}$ are the characteristic exponents for $\tilde{\pi}\left(\gamma_{1}\right)$. Define measurable distributions $\mathcal{W}_{k}$ on $M \times \mathbf{H}_{\mathbb{R}} /\left(\mathbf{H}^{0}\right)_{\mathbb{R}}$ by $\mathcal{W}_{k}=\sigma_{0}(x,[h]) \bar{\sigma}(x,[h]) W_{k}$. Lemma 2.2 shows that for the maximal characteristic exponent $\chi_{1}$ of $\tilde{\pi}\left(\gamma_{1}\right)$, the corresponding distribution $\mathcal{W}_{1}=$ $E_{1}$ almost everywhere and hence coincides almost everywhere with a continuous distribution.

Let $p_{1}=W_{1} \in \mathbf{P} \mathbb{R}^{n}, \eta_{1}=I d$ and $\eta_{2}, \ldots, \eta_{l} \in \Gamma$. Let $p_{k}=\tilde{\pi}\left(\eta_{k}\right) p_{1}$. It is easy to see that $p_{k}$ is the eigenspace corresponding to $\tilde{\pi}\left(\eta_{k} \eta_{1} \eta_{k}^{-1}\right)$. Thus $\bar{\sigma}\left(x,[h] p_{k}\right)$ coincides almost everywhere with the lift of 1-dimensional strongest stable distribution for $\rho\left(\eta_{k} \eta_{1} \eta_{k}^{-1}\right)$ to $M \times \mathbf{H}_{\mathbb{R}} /\left(\mathbf{H}^{0}\right)_{\mathbb{R}}$. We define a map $\Phi: M \times \mathbf{H}_{\mathbb{R}} /\left(\mathbf{H}^{0}\right)_{\mathbb{R}} \rightarrow\left(\mathbf{P} \mathbb{R}^{n}\right)^{l}$, $(x,[h]) \mapsto\left(\bar{\sigma}(x,[h]) p_{1}, \ldots, \bar{\sigma}(x,[h]) p_{l}\right)$. We remark that this map is independent of $[h]$ and coincides almost everywhere with a continuous map. iff

Consider the map $\Psi: \mathbf{P G L}(n, \mathbb{R}) \rightarrow\left(\mathbf{P} \mathbb{R}^{n}\right)^{l}, \bar{g} \mapsto\left(\bar{g} p_{1}, \ldots, \bar{g} p_{l}\right) . \Psi$ is injective

$$
\bigcap_{k=1}^{l} \mathbf{P G L}(n, \mathbb{R})_{p_{k}}=\{\operatorname{Id}\} .
$$

We now show that we can choose $\eta_{k}$ such that $\Psi$ is indeed injective. We observe that the intersection $T=\bigcap_{\gamma \in \Gamma} \mathbf{P G L}(n, \mathbb{R})_{\tilde{\pi}(\tilde{\gamma}) p_{1}}=\bigcap_{\gamma \in \Gamma} \tilde{\pi}(\tilde{\gamma})\left(\mathbf{P G L}(n, \mathbb{R})_{p_{1}}\right) \tilde{\pi}(\tilde{\gamma})^{-1}$ fixes the images of $p_{1}$ under $\tilde{\pi}(\tilde{g})$ for all $\tilde{g} \in\left(\tilde{\mathbf{G}}_{\mathbb{R}}\right)^{0}, p_{1}$ is one-dimensional, and $\tilde{\pi}$ is irreducible. Therefore for a suitable basis (of the form $\left\{\tilde{\pi}\left(\tilde{\eta}_{i}\right) p_{1}\right\}_{i=1, \ldots, n}$ for some $\eta_{i} \in \Gamma$; we fix this basis for the rest of the proof), each element in $T$ is diagonal and hence $T$ is abelian. Moreover $T$ is normalized by $\tilde{\pi}(\tilde{\gamma})$ for all $g \in \Gamma$ hence for all $\tilde{g} \in\left(\tilde{\mathbf{G}}_{\mathbb{R}}\right)^{0}$. We now show that $T$ is trivial. Otherwise let $A \in T$ such that the lift of $A$ to $S L(n, \mathbb{R})$ (still denoted by $A$ ) has the form $A=a_{1} I d_{\mathbb{R}^{n_{1}}} \oplus \cdots \oplus a_{k} I d_{\mathbb{R}^{n_{k}}}$, $a_{i} \in \mathbb{R}, k \geq 2$ and $a_{i} \neq a_{j}$ if $i \neq j$. It is clear that $A$ stabilizes the spaces $\mathbb{R}^{n_{i}}$. Since $\tilde{\pi}\left(\left(\tilde{\mathbf{G}}_{\mathbb{R}}\right)^{0}\right)$ is connected, for any neighborhood $U$ of $e \in \tilde{\pi}\left((\tilde{\mathbf{G}})_{\mathbb{R}}\right), U$ generates $\tilde{\pi}(\tilde{G})$. Since $T$ is normalized by $U$, there exists $A^{\prime}(u) \in T$ such that $u A u^{-1}=A^{\prime}(u)$ for every $u \in U$. Spell it out in terms of matrices with respect the fixed basis; it follows easily that if $U$ is small enough, then $A^{\prime}(u)=A$ for all $u \in U$. So it follows that $u A=A u$ and $u$ stabilizes $\mathbb{R}^{n_{i}}$ also. Therefore, $\tilde{\pi}\left(\left(\tilde{\mathbf{G}}_{\mathbb{R}}\right)^{0}\right)$ stabilizes $\mathbb{R}^{n_{i}}$, contrary to the irreducibility of $\tilde{\pi}$.

Since $T$ is trivial, we may choose $\eta_{1}, \ldots, \eta_{l}$ such that

$$
\bigcap_{k=1}^{l} \mathbf{P G L}(n, \mathbb{R})_{p_{k}}=\{I d\} .
$$

Therefore, $\Psi$ is injective. Moreover, we may choose them so that $l \geq n+1$ and any $n$ of elements in $\left\{p_{1}, \ldots, p_{n+1}\right\}$ spans $\mathbb{R}^{n}$ (and hence for any $\bar{g} \in \mathbf{P G L}(n, \mathbb{R})$, 
any $n$ of elements in $\left\{\bar{g} p_{1}, \ldots, \bar{g} p_{n+1}\right\}$ spans $\left.\mathbb{R}^{n}\right)$. Indeed, we may choose $p_{1}, \ldots, p_{n}$ spanning $\mathbb{R}^{n}$. For any $n-1$ elements $\left\{p_{i_{1}}, \ldots, p_{i_{n-1}}\right\}$ of $\left\{p_{1}, \ldots, p_{n}\right\},\{\tilde{g} \in \tilde{\mathbf{G}}$ : $\tilde{\pi}(\tilde{g}) p_{1}$ and $p_{i_{1}}, \ldots, p_{i_{n-1}}$ span $\left.\mathbb{R}^{n}\right\}$ is a non-empty Zariski-open set. Since the intersection of any finite Zariski open sets is non-empty, there exists $\tilde{\gamma} \in \tilde{\Gamma}$ such that $p_{n+1}:=\tilde{\pi}(\tilde{\gamma}) p_{1}$ together with any $n-1$ elements in $\left\{p_{1}, \ldots, p_{n}\right\}$ spans $\mathbb{R}^{n}$ by the Borel density theorem. Let $U$ be the image of $\Psi$ with the induced subspace topology. We show that $U$ and $\mathbf{P G L}(n, \mathbb{R})$ are homeomorphic. It is sufficient to show that the inverse of $\Psi$ is continuous. Let $u_{k}=\left(u_{1}^{(k)}, \ldots, u_{l}^{(k)}\right) \in U$ and $u_{k} \rightarrow u_{0}=\left(u_{1}^{(0)}, \ldots, u_{l}^{(0)}\right) \in U$. Let $g_{k} \in \mathbf{G L}(n, \mathbb{R})$ so that $\Psi\left(\bar{g}_{k}\right)=u_{k}$. By multiplying a scalar matrix we may assume $\left\|g_{k}\right\|(k=1,2, \ldots)$ are bounded and bounded away from zero matrix. Take any convergent sequence $g_{k_{j}} \rightarrow g_{0} \neq 0$. We show that $g_{0} \in G L(n, \mathbb{R})$. Otherwise, by the compactness of $\mathbf{P} \mathbb{R}^{n}$ and by passing to a subsequence we may assume that $\lim \Psi\left(\bar{g}_{k_{j}}\right)=\lim \left(\bar{g}_{k_{j}} p_{1}, \ldots, \bar{g}_{k_{j}} p_{l}\right)=\left(p_{1}^{\prime}, \ldots, p_{l}^{\prime}\right)=u_{0}$. Making use of an elegant trick due to Furstenburg (see for example the proof of 3.2 .1 in [Z2]) we conclude that for all $k=1, \ldots, l, p_{k}^{\prime}$ is either in the image $\operatorname{im}\left(g_{0}\right)$ of $g_{0}$ or in the kernel $\operatorname{ker}\left(g_{0}\right)$ of $g_{0}$. Let $\operatorname{dim}\left(\operatorname{im}\left(g_{0}\right)\right)=n_{1}$ and $\operatorname{dim} \operatorname{ker}\left(g_{0}\right)=n_{2}$; then $n_{1}+n_{2}=n$. So in $p_{1}^{\prime}, \ldots, p_{n+1}^{\prime}$, either at least $n_{1}+1$ elements of them are in $\operatorname{im}\left(g_{0}\right)$ or at least $n_{2}+1$ elements of them are in $\operatorname{ker}\left(g_{0}\right)$. So we conclude that some $n$ elements of $\left(p_{1}^{\prime}, \ldots, p_{n+1}^{\prime}\right)$ no longer span $\mathbb{R}^{n}$ and hence $\left(p_{1}^{\prime}, \ldots, p_{l}^{\prime}\right) \neq u_{0}=$ $\left(u_{1}^{(0)}, \ldots, u_{l}^{(0)}\right)$ since any $n$ elements of $\left\{u_{1}^{(0)}, \ldots, u_{n+1}^{(0)}\right\}$ span $\mathbb{R}^{n}$. This contradiction shows that $g_{0} \in G L(n, \mathbb{R})$. We next show that $\bar{g}_{k}$ converges. For any two converging sequences $g_{k_{j_{1}}} \rightarrow g_{1} \in G L(n, \mathbb{R})$ and $g_{k_{j_{2}}} \rightarrow g_{2} \in G L(n, \mathbb{R})$, we have $\lim \Psi\left(\bar{g}_{k_{j_{1}}}\right)=\left(\bar{g}_{1} p_{1}, \ldots, \bar{g}_{1} p_{l}\right)=u_{0}$ and $\lim \Psi\left(\bar{g}_{k_{j_{2}}}\right)=\left(\bar{g}_{2} p_{1}, \ldots, \bar{g}_{2} p_{l}\right)=u_{0}$. Since $\Psi$ is injective, $\bar{g}_{1}=\bar{g}_{2}$.

Therefore, $\Psi^{-1}: \mathbf{P G L}(n, \mathbb{R}) \rightarrow U$ is continuous and hence $\Psi$ is a homeomorphism.

Observe that $\bar{\sigma}(x,[h])$ coincides with $\Psi^{-1}(\Phi(x,[h]))$ almost everywhere, and $\Phi$ is independent of $[h]$; we obtain that $\bar{\sigma}(x,[h])$ coincide with a lift of a continuous framing on $M$ (still denoted by $\bar{\sigma}(x))$ almost everywhere.

We remark that since $\rho$ is orientable weakly Cartan, the distributions $E_{i}$ are Hölder distributions ([BP]). Hence $\bar{\sigma}(x)$ is easily seen to be Hölder from our proof, and we may find a Hölder framing $\sigma_{*}$ of $T M$ projecting to $\bar{\sigma}(x)$.

Proof of Theorem 1.1. Let $\sigma_{*}(x)=f(x,[h]) \sigma(x,[h])$ for a real valued function $f$. We have

$$
\begin{aligned}
D \rho(\gamma) \sigma_{*}(x) & =f(x,[h]) D \rho(\gamma) \sigma(x,[h])=f(x,[h]) \sigma\left(\rho^{\prime}(\gamma)(x,[h])\right) \tilde{\pi}(\tilde{\gamma}) b(x,[h], \tilde{\gamma}) \\
& =f(x,[h]) f^{-1}\left(\rho^{\prime}(\gamma)(x,[h])\right) \sigma_{*}(\rho(\gamma) x) \tilde{\pi}(\tilde{\gamma}) b(x,[h], \tilde{\gamma}) \\
& =\sigma_{*}(\rho(\gamma) x) \tilde{\pi}(\tilde{\gamma}) g(x,[h], \tilde{\gamma}),
\end{aligned}
$$

for a function $g$ taking values in an abelian group $A \cong \mathbb{R}^{+} \times\left(\bigoplus_{i=1}^{k}\left\{ \pm \operatorname{Id}_{V_{i}}\right\}\right)$ centralizing the image of $\tilde{\pi}$. It is obvious that $g$ is independent of $[h]$, and coincides with a Hölder continuous function (still denoted by $g$ ) almost everywhere. $g$ is clearly an $A$-valued cocycle of $\tilde{\Gamma}$ over the action $(\tilde{\gamma}, x) \mapsto \rho(\gamma) x$ for $\tilde{\gamma} \in \tilde{\Gamma}(\tilde{\gamma}$ projects to $\gamma \in \Gamma)$. Since $g$ is continuous and clearly non-vanishing, the projection of $g$ to the second factor of $A$ is constant; thus we obtain a homomorphism of $\tilde{\Gamma}$ to $Z=\bigoplus_{i=1}^{k}\left\{ \pm \operatorname{Id}_{V_{i}}\right\}$. Therefore, we may take a subgroup $\tilde{\Gamma}_{1} \subset \tilde{\Gamma}$ of finite index (the 
index is less than $\left.2^{n}\right)$, such that the projection to the second factor of $g(x, \tilde{\gamma})$ is the identity matrix. So when $g$ is considered as a cocycle of $\tilde{\Gamma}_{1}$, it is $\mathbb{R}^{+}$-valued. By 9.1.1 of [Z2], $g$ is equivalent to the trivial cocycle (i.e., there exists a measurable $\mathbb{R}^{+}$-valued function $p$, such that $\left.g(x, \tilde{\gamma})=p^{-1}(x) p(\rho(\gamma) x)\right)$. By an argument of Livsic ( $\S 6$ of $[\mathrm{Li}])$ and the absolute continuity of the strongest stable foliations ( 33 of $[\mathrm{BP}]$ ), we conclude that $p$ coincides with a Hölder continuous function (still denoted by $p$ ). It is clear that when restricted to $\Gamma_{1}, \tilde{\pi}(\tilde{\gamma})$ depends only on the projection $\gamma$ of $\tilde{\gamma}$. We denote it by $\pi(\gamma)$. Denote by $\Gamma_{0}$ the projection of $\tilde{\Gamma}_{1}$ to $\Gamma$. Then $\left.\rho\right|_{\Gamma_{0}}$ is Hölder tangentially flat with respect to a Hölder continuous framing $p(x) \sigma_{*}(x)$ and homomorphism $\pi$.

Remark. (a) If in addition $\mathbf{G}$ is algebraically simply connected, we may take $\tilde{\Gamma}=\Gamma$. If there exist $k \geq 2$ irreducible constituents for $\tilde{\pi}(\tilde{\Gamma})$, we need to restrict $\rho$ to a subgroup $\Gamma_{0}$ of index $\leq 2^{k+1}<2^{n}$, so that $\left.\rho\right|_{\Gamma_{0}}$ is Hölder tangentially flat. From the proof it is clear that the corresponding homomorphism $\left.\pi\right|_{\Gamma_{0}}$ extends to a continuous homomorphism from $G \rightarrow G L(n, \mathbb{R})$.

(b) If the smoothness class of $\rho$ in Theorem 1.1 is weakened to be only $C^{1}$, and the invariant probability measure $m$ has positive continuous density, then $\rho$ is $C^{0}$ tangentially flat if $\rho$ is orientation-preserving. If $\rho$ is not orientation-preserving, $\rho$ is $C^{0}$-tangentially flat when restricted to a subgroup of index 2 . The proof is essentially the same. We indicate the necessary changes.

Let $\sigma_{0}$ be a continuous framing with unit volume everywhere. Then the derivative cocycle of $\rho$ takes values in $S L(n, \mathbb{R})$ (when restricted to a subgroup $\Gamma^{\prime}$ of index 2 if $\rho$ is not orientation-preserving). Then $\sigma$ takes values in $S L(n, \mathbb{R})$ and $\bar{\sigma}$ takes values in $\operatorname{PSL}(n, \mathbb{R})$. Lift $\bar{\sigma}$ to a continuous framing $\sigma_{*}$ taking values in $S L(n, \mathbb{R})$. Then $g(x,[h], \tilde{\gamma})$ as in the proof of Theorem 1.1 coincides with a continuous function and has determinant 1 . Thus it is necessarily independent of $(x,[h])$ (denoted by $g(\tilde{\gamma}))$, and hence a homomorphism to $\bigoplus_{i=1}^{k}\left\{ \pm \operatorname{Id}_{V_{i}}\right\}$. Therefore it is easy to see that $\sigma_{*}$ is

a linearizing framing for $\rho$ with homomorphism $\tilde{\pi}(\tilde{\gamma}) g(\tilde{\gamma})$ (for the subgroup $\tilde{\Gamma}^{\prime}$ if $\rho$ is not orientation-preserving) that clearly factor through to a homomorphism from $\Gamma$ ( $\Gamma^{\prime}$ if $\rho$ is not orientation-preserving) to $S L(n, \mathbb{R})$.

(c) The functions $\Phi$ and $\Psi$ in Lemma 2.3 are first considered by Katok, Lewis and Zimmer [KLZ]. We state the following result. Assume that $\sigma: M \rightarrow \mathbf{P G L}(n, \mathbb{R})$ is continuous $(n=\operatorname{dim}(M)), p_{1}, \ldots, p_{l}$ be $l$ elements in Grassmannian $\operatorname{Gr}\left(n_{1}, n\right)$ of $n_{1}$-planes $\left(1 \leq n_{1} \leq n-1\right)$ such that $\bigcap_{k=1}^{l} \mathbf{P G L}(n, \mathbb{R})_{p_{k}}=\{\operatorname{Id}\}$. If for each $k=1, \ldots, l, \sigma(x) p_{k}$ is a $C^{r}$ distribution in $T M$, then $\bar{\sigma}(x)$ is $C^{r}$. It is clear that $\Psi$ is a one to one map from $\operatorname{Gr}\left(n_{1}, n\right)$ to the image of $\Psi$. We then use the inverse function theorem to conclude that $\Psi$ is a local $C^{\infty}$ diffeomorphism. We refer the reader to [KLZ] for details.

(d) From (c) above, to obtain a $C^{r} \bar{\sigma}$, it is sufficient to obtain certain $C^{r}$ distributions for (partially) hyperbolic elements. The regularity of such distributions is discussed by Brin, Pesin [BP], and Hirsch, Pugh [HP].

\section{Local Lie group structure}

We first show that under the conditions in Theorem 1.2, the linearizing $C^{1}$ framing $\sigma(x)$, which can be viewed as a set of $n$ non-vanishing $C^{1}$ vector fields, generates a finite dimensional Lie algebra. 
Let $X_{1}, \ldots, X_{n}$ be $n$ non-vanishing $C^{1}$-vector fields on $M$; they determine a trivialization for the tangent bundle $T M$. For any $x \in M$, we identify $T_{x} M$ naturally with $\mathbb{R}^{n}$ by the trivialization (i.e., for each $i=1, \ldots, n$, we identify $e_{i} \in \mathbb{R}^{n}$ with $\left.X_{i}(x) \in T_{x} M\right)$ and define a map $f(x): \mathbb{R}^{n} \times \mathbb{R}^{n} \rightarrow \mathbb{R}^{n}$ as follows. Let $u, v \in \mathbb{R}^{n}=$ $T_{x} M$, and $u=\left(u_{1}, \ldots, u_{n}\right), v=\left(v_{1}, \ldots, v_{n}\right)$. Let $\left[\sum_{k=1}^{n} u_{k} X_{k}, \sum_{k=1}^{n} v_{k} X_{k}\right](x)=$ $\sum_{k=1}^{n} w_{k} X_{k}(x)$. Then $f(x)(u, v)=\left(w_{1}, \ldots, w_{n}\right)$. It is clear that $f(x)$ is a bilinear map from $\mathbb{R}^{n} \times \mathbb{R}^{n}$ to $\mathbb{R}^{n}$. Let $B L(n)$ be the linear space containing all bilinear maps from $\mathbb{R}^{n} \times \mathbb{R}^{n}$ to $\mathbb{R}^{n}$. For $\pi: \Gamma \rightarrow G L(n, \mathbb{R})$ we may define a homomorphism $H: \Gamma \rightarrow G L(B L(n))$ by the rule

$$
(H(\gamma) g)(u, v)=\pi(\gamma) g\left(\pi\left(\gamma^{-1}\right) u, \pi\left(\gamma^{-1}\right) v\right)
$$

for all $g \in B L(n)$. It is easy to see that $f(\rho(\gamma) x)=(H(\gamma) f)(x)$.

The following lemma was pointed out to me by Margulis. It considerably improves an earlier version of the lemma.

Lemma 3.1. Let $m$ be an invariant measure on $M, \rho$ be a m-preserving action of $\Gamma$ on $M, H: \Gamma \rightarrow G L(N, \mathbb{R})(N \geq 1)$ be a homomorphism which extends to a homomorphism from $G$ to $G L(N, \mathbb{R})$, and $F: M \rightarrow \mathbb{R}^{N}$ be a measurable map. Assume $F(\rho(\gamma) x)=(H(\gamma) F)(x)$ for almost all $x \in M$. Then $H(\gamma) F(x)=F(x)$ for all $\gamma \in \Gamma$ and almost all $x \in M$.

Proof. Without loss of generality, we assume that $m$ is ergodic. It is easy to see that $\Gamma$ acts on $\mathbb{R}^{N}$ via $H: \gamma \circ v=H(\gamma) v$. We define a measure $m^{\prime}$ on $\mathbb{R}^{N}$ by $m^{\prime}(A)=$ $m\left(F^{-1} A\right)$ for all measurable sets $A \in \mathbb{R}^{N}$. For any such $A$ it is easy to verify that $F^{-1}\left(H\left(\gamma^{-1}\right) A\right)=\rho\left(\gamma^{-1}\right) F^{-1}(A)$ and hence $m^{\prime}\left(\gamma^{-1} A\right)=m\left(F^{-1}\left(H\left(\gamma^{-1}\right) A\right)\right)=$ $m\left(\rho\left(\gamma^{-1}\right) F^{-1}(A)\right)=m\left(F^{-1}(A)\right)$. So $m^{\prime}$ is invariant under $\Gamma$ and ergodic. Observe that either $m^{\prime}(\{0\})=1$ (in this case $F(x)=0$ for $m$-almost all $x \in M$ and our lemma follows); or $m^{\prime}(\{0\})=0$ (in this case $F(x) \neq 0$ for $m$-almost all $x \in M$ ). We assume without loss of generality that $F(x) \neq 0$. Consider the induced action $\rho_{1}$ of $\Gamma$ on $\mathbf{P} \mathbb{R}^{N}$ via the projection $\operatorname{proj}$ of $H(\gamma)$ to $\mathbf{P G L}(N, \mathbb{R})$. Then $m^{\prime \prime}=(p r o j)_{*} m^{\prime}$ is a $\rho_{1}$-invariant ergodic measure on $\mathbf{P G L}(N, \mathbb{R})$ and again ergodic.

Since $G$ is connected, semisimple and $G$ has no non-trivial compact factor, for any irreducible constituent $H_{i}$ of $H H_{i}(G) \subset S L(N, \mathbb{R})$ and $H(\Gamma)$ is not precompact unless $H_{i}$ is trivial.

We now show that $H$ is essentially trivial by induction on $N$. If $N=1, H$ trivial since there exists no nontrivial representation of $G$ on $\mathbb{R}$. Consider any $N \geq 2$. From Lemma 3.2.1 and Corollary 3.2.2 of [Z2] we see that either $H(\Gamma) /\{ \pm I d\}$ is precompact in $\mathbf{P G L}(N, \mathbb{R})$, in this case $H(\Gamma)$ is precompact in $S L(N, \mathbb{R})$ which implies that $H$ is trivial (since every nontrivial continuous representation of $G$ on $\mathbb{R}^{N}$ is non-compact), or there exists a proper subspace $V_{0} \subset \mathbb{R}^{N}$ such that $m^{\prime \prime}$ is supported in a finite union of proper projective subspaces $\left[V_{0}\right], \ldots,\left[V_{s}\right]$ (which is the orbit of $\left[V_{0}\right]$ under the subgroup $\mathbf{P G L}(N, \mathbb{R})_{m^{\prime \prime}}$ of $\mathbf{P G L}(N, \mathbb{R})$ keeping $m^{\prime \prime}$ invariant) and for a subgroup $\Gamma_{1} \subset \Gamma$ of finite index, $\Gamma_{1}$ fixes $\left[V_{0}\right]$. It is easy to see that $V_{0}$ is also invariant under $H$ (by the nature of $H$ ) and hence $s=1$. Now the restricted action of $\Gamma$ on $\left[V_{0}\right]$ preserves an invariant measure $m^{\prime \prime \prime}=\left.m^{\prime \prime}\right|_{\left[V_{0}\right]}$. Since $m^{\prime \prime}$ is ergodic, and $m^{\prime \prime \prime}<<m^{\prime \prime}, m^{\prime \prime \prime}=m^{\prime \prime}$. Hence we conclude that the restriction of the action of $\rho_{1}$ of $\Gamma$ on $\left[V_{0}\right]$ preserves an invariant ergodic measure $m^{\prime \prime}$. By induction, $\left.H\right|_{V_{0}}$ is essentially trivial. Or for $m^{\prime}$-almost all $v \in V_{0}, H v=v$. So for $m$-almost every $x \in M, H(\gamma) F(x)=F(x)$ for all $\gamma \in \Gamma$. 
Corollary 3.2. Let $\rho$ be as in Theorem $1.2, \sigma(x)=\left\{X_{1}, \ldots, X_{n}\right\}$ be the $C^{1}$ linearizing framing. Then the linear vector space generated by $X_{1}, \ldots, X_{n}$ has a Lie algebra structure.

Proof. Let $\pi$ be as in Theorem 1.2. Let $f$ be a bilinear map defined as before. Then we have $f(\rho(\gamma) x)=(H(\gamma) f)(x)$ for some homomorphism $H$ from $\Gamma$ to the space $B L(n)$ of bilinear maps from $\mathbb{R}^{n} \times \mathbb{R}^{n}$ to $\mathbb{R}^{n}$ which extends to a homomorphism of $G$ (because $\pi$ does). By Lemma 3.1, for almost all $x \in M, f(\rho(\gamma) x)=f(x)$. Since $\rho$ is ergodic and $f$ is continuous, $f$ is constant. Or, equivalently, if $x_{0} \in M$ and $\left[X_{i}, X_{j}\right]\left(x_{0}\right)=\sum_{k=1}^{n} C_{i j}^{k} X_{k}\left(x_{0}\right)$ for some constants $C_{i j}^{k}$, then $\left[X_{i}, X_{j}\right](x)=$ $\sum_{k=1}^{n} C_{i j}^{k} X_{k}(x)$ for all $x \in M$. So $\left[X_{i}, X_{j}\right]=\sum_{k=1}^{n} C_{i j}^{k} X_{k}$.

Now we recall a result concerning the local Lie group structure for a smooth manifold. Suppose there exist $n$ non-vanishing smooth vector fields on an $n$-dimensional smooth manifold so that they form an $n$-dimensional Lie algebra under the Lie bracket. It is a classic result that $M$ has a local Lie group structure (see for example, [W]). Although $C^{\infty}$-smoothness is assumed in [W], it is clear that the argument goes through if the vector fields are of class $C^{2}$. With a slight modification of the argument, we can prove that the $C^{1}$ smoothness of the vector fields is enough to ensure the local Lie group structure on $M$.

We state the above as follows.

Lemma 3.3. Let $M$ and $N$ be smooth manifolds of dimension $n,\left\{X_{i}\right\}_{i=1}^{n}$ and $\left\{Y_{i}\right\}_{i=1}^{n}$ be $C^{r}(r \geq 1)$ framings on $M$ and $N$, respectively. Assume that $\left[X_{i}, X_{j}\right]=$ $\sum_{k=1}^{n} C_{i j}^{k} X_{k}$ and $\left[Y_{i}, Y_{j}\right]=\sum_{k=1}^{n} C_{i j}^{k} Y_{k}$, where $C_{i j}^{k}$ are constants. Then for every $m \in M$ and $n \in N$ there exist neighborhoods $U$ of $m$ and $V$ of $n$ and $a C^{r+1}$ diffeomorphism $F: U \rightarrow V$ such that $F_{*} X_{i}=Y_{i}$.

Proof. If $r \geq 2$, it is essentially proved in [W]; see also Lemma 3.4 of [F]. If $r=1$ and $C_{i j}^{k}=0$, see also Lemma 3.4 of $[\mathrm{F}]$. In general for $r=1$, we will give a proof in $\S 5$. (In fact, the proof in $\S 5$ applies to all cases $r \geq 1$.)

We recall an argument of Feres $[\mathrm{F}]$. Let $L$ be a connected, simply connected Lie group with Lie algebra $\mathcal{L}$ isomorphic to the Lie algebra generated by $\left\{X_{i}\right\}_{i=1}^{n}$. It follows from Lemma 3.3 that $M$ admits a $C^{r+1}(L, L)$-structure in the sense of Thurston $[\mathrm{T}]$ which is complete (Proposition 3.6 of $[\mathrm{T}]$ ). Consequently, we obtain a $C^{r+1}$-diffeomorphism between the universal cover of $M$ and $L$ that maps the lift of $X_{i}$ to a right-invariant vector field on $L$. The deck transformations of $\tilde{M}$ are conjugate under the diffeomorphism to a group $\Lambda$ of affine automorphisms of $L$ that preserve the vector fields $X_{i}$. In other words, we have the following.

Corollary 3.4. Let $M$ be a smooth compact manifold and there is a $C^{r}$-framing $\sigma(x)=\left\{X_{1}, \ldots, X_{n}\right\}$ such that the linear vector space generated by $X_{1}, \ldots, X_{n}$ has a Lie algebra structure. Denote the Lie algebra by $\mathcal{L}$ and the corresponding connected, simply connected Lie group by L. Let $\rho$ be a tangentially flat action of $\Gamma$ on $M$ with respect to $\sigma$. Then there exist a cocompact lattice $\Lambda$ in $L$, a homomorphism $\rho_{0}: \Gamma \rightarrow \operatorname{Aff}(L / \Lambda)$, a $C^{r+1}$-diffeomorphism $\phi: M \rightarrow L / \Lambda$, such that $\rho(\gamma)=\phi^{-1} \rho_{0}(\gamma) \phi$.

Proof of Theorem 1.2. Under the condition our action $\rho$ is tangentially linear, ergodic and the linearizing framing $\left\{X_{1}, \ldots, X_{n}\right\}$ is $C^{r}(r \geq 1)$; hence there is a Lie algebra structure for the vector space spanned by vector fields $\left\{X_{1}, \ldots, X_{n}\right\}$ (Lemma 3.2). Our theorem follows from Corollary 3.4. 


\section{Applications}

In Theorem 1.1 we showed that an orientable weakly Cartan action is $C^{0}$ tangentially flat. The linearizing framing we obtained is only continuous. By Theorem 1.2, to obtain $C^{2}$ global rigidity we need at least the $C^{1}$-smoothness of the framing. In some special cases, we have such smoothness. We present an example below. For more examples, see [Q2].

Let $\mathcal{A}$ be a free Abelian group and $\rho: \mathcal{A} \rightarrow \operatorname{Diff}^{r}(M)$ be an action of $\mathcal{A}$ on $M$. $\rho$ is said to be a $C^{r}$-Cartan action on the manifold $M$ if (a) There exists a set of elements $\Delta=\left\{\gamma_{1}, \ldots, \gamma_{n}\right\} \subset \mathcal{A}$ such that for each $\gamma_{i} \in \Delta, \rho\left(\gamma_{i}\right)$ is Anosov and has a 1-dimensional, strongest stable distribution $E_{i}^{s s}$; and (b) the distributions $E_{i}^{s s}$ are pairwise-transversal with their internal direct sum $E_{1}^{s s} \oplus \cdots \oplus E_{n}^{s s} \cong T M$. For an action $\rho$ of $\Gamma$ on $M$, if there exists a free abelian group $\mathcal{A} \subset \Gamma$, such that the restriction $\left.\rho\right|_{\mathcal{A}}$ of $\rho$ to $\mathcal{A}$ is a Cartan action of $\mathcal{A}$ on $M$, we say that $\rho$ itself is a Cartan action of $\Gamma$ on $M$. Cartan actions are first considered by Hurder [Hu1]. It is clear that a Cartan action is a weakly Cartan action. We recall Hurder's global rigidity for abelian Cartan actions.

Theorem 4.1 [Hu1] [Hu2]. Let $\phi$ be a Cartan $C^{r}$-action of an abelian group $\mathcal{A}$ on an infra-nilmanifold $M$, for $r=1, \infty$ or $\omega$. If $\phi$ has constant exponents, then there is a subgroup $\tilde{\mathcal{A}} \subset \mathcal{A}$ of finite index so that the restriction $\left.\phi\right|_{\tilde{\mathcal{A}}}$ is $C^{r}$-conjugate to a standard linear action, and $\phi$ is $C^{r}$-conjugate to an affine action $\phi_{*}$ of $\mathcal{A}$ on $M$.

The global rigidity of higher rank lattice Cartan actions on infra-nilmanifolds follows from the tangential flatness of such actions and Theorems 1.2, 4.1.

Theorem 4.2. Let $\rho$ be a Cartan $C^{r}$-action of $\Gamma$ on an infra-nilmanifold $M$ with a finite orbit (i.e., there exists $p \in M$ such that $\#(\rho(\Gamma) p)<\infty)$, for $r=1, \infty$ or $\omega$. Then $\left.\rho\right|_{\Gamma_{0}}$ is $C^{r}$-conjugate to an affine action of $\Gamma_{0}$ on $M$ for a subgroup $\Gamma_{0} \subset \Gamma$ of finite index.

Proof. Since $\#(\rho(\Gamma) p)$ is finite, $\rho$ induces an action on the orbit $\rho(\Gamma) p$ of $p$. Therefore there exists a subgroup $\Gamma_{1} \subset \Gamma$ of finite index such that $\rho\left(\Gamma_{1}\right) p=p$. Let the connected nilmanifold $\tilde{M}$ cover $M$, pr $: \tilde{M} \rightarrow M$ the covering map. We fix $\tilde{p} \in \tilde{M}$ such that $\tilde{p}$ projects to $p$.

For any $\sigma \in \pi_{1}(\tilde{M}, \tilde{p})$, we choose a representative $\phi_{\sigma}:[0,1] \rightarrow \tilde{M}$ with base point $\phi_{\sigma}(0)=\phi_{\sigma}(1)=\tilde{p}$. For $\gamma \in \Gamma_{1}, \rho(\gamma) \circ \operatorname{pr} \circ \phi_{\sigma}$ is a path in $M$. We fix $q \in \operatorname{pr}^{-1} p$ and lift this path to $\psi_{q, \gamma, \sigma}$ so that the initial point is $q$. Let the end point of the lifted path be $r=\psi_{q, \gamma, \sigma}(1)$. It is clear that $r$ only depends on $\sigma, \gamma$ and $q$. In other words, for fixed $\sigma \in \pi_{1}(\tilde{M}, \tilde{p}),\left.\rho\right|_{\Gamma_{1}}$ induces an action $\rho_{\sigma}$ of $\Gamma_{1}$ on $\operatorname{pr}^{-1} p$ by $\rho_{\sigma}(\gamma)(q)=r$, $q, r$ as above. Consequently, there exists a subgroup $\Gamma_{\sigma} \subset \Gamma_{1}$ of finite index, such that for each $\gamma \in \Gamma_{\sigma}, \rho_{\sigma}(\gamma)(q)=q$ for all $q \in \operatorname{pr}^{-1} p$.

Since $\tilde{M}$ is a nilmanifold, $\pi_{1}(\tilde{M}, \tilde{p})$ is finitely generated. Let $\sigma_{1}, \ldots, \sigma_{d}$ be the generators. For $\sigma_{1}$, there exists a subgroup $\Gamma_{\sigma_{1}} \subset \Gamma_{1}$ of finite index, such that for each $\gamma \in \Gamma_{\sigma_{1}}, \rho_{\sigma_{1}}(\gamma)(q)=q$ for all $q \in \operatorname{pr}^{-1} p$. For $\sigma_{2}$, there exists a subgroup $\Gamma_{\sigma_{2}} \subset \Gamma_{\sigma_{1}}$ of finite index, such that for each $\gamma \in \Gamma_{\sigma_{2}}, \rho_{\sigma_{2}}(\gamma)(q)=q$ for all $q \in \mathrm{pr}^{-1} p$. Continuing in this way step by step, we see that there exists a subgroup of finite index $\Gamma_{*} \subset \Gamma$, such that for all $i=1, \ldots, d, \gamma \in \Gamma_{*}, q \in \operatorname{pr}^{-1} p, \rho_{\sigma_{i}}(\gamma)(q)=q$. Consequently, for all $\sigma \in \pi_{1}(\tilde{M}, \tilde{p}), \gamma \in \Gamma_{*}, q \in \operatorname{pr}^{-1} p, \rho_{\sigma}(\gamma)(q)=q$.

Now it is clear that for each $\gamma \in \Gamma_{*}$, we can lift $\rho(\gamma)$ to a diffeomorphism $\tilde{\rho}(\gamma)$ such that $\tilde{\rho}(\gamma)(\tilde{p})=\tilde{p}$. It can be verified easily that $\tilde{\rho}$ is an action of $\Gamma_{*}$ on $\tilde{M}$ that covers $\left.\rho\right|_{\Gamma_{*}}$. 
We may assume that $\tilde{\rho}$ is orientable Cartan action. Since $\left.\tilde{\rho}\right|_{\Gamma_{*}}$ is tangentially flat by Theorem 1.1, there exist a continuous framing $\sigma$ on $\tilde{M}$, a subgroup $\Gamma_{0} \subset \Gamma_{*}$ of finite index, a homomorphism $\pi: \Gamma_{0} \rightarrow G L(n, \mathbb{R})$, such that the derivative cocycle of $\left.\tilde{\rho}\right|_{\Gamma_{0}}$ is given by $\pi$. By a $C^{r}$-conjugacy $\tilde{F}$ (which is a lift of the conjugacy $F$ from Theorem 4.1) if necessary, we may assume that $\sigma$ is $C^{\infty}$ and $\sigma$ consists of right invariant vector fields on the nilmanifold $\tilde{M}$. Hence by $C^{r}$ conjugacy $\tilde{F}$, $\left.\tilde{F} \tilde{\rho}\right|_{\Gamma_{0}}(\gamma) \tilde{F}^{-1}$ is an affine diffeomorphism on $\tilde{M}$ for every $\gamma \in \Gamma_{0}$.

Since $\tilde{F}$ is the lift of the conjugacy for the abelian Cartan action (from Theorem 4.1), the diffeomorphism $\left.\tilde{F} \tilde{\rho}\right|_{\Gamma_{0}}(\gamma) \tilde{F}^{-1}$ on $\tilde{M}$ factors through to a diffeomorphism $\left.F \rho\right|_{\Gamma_{0}}(\gamma) F^{-1}$ on $M$ for every $\gamma \in \Gamma_{0}$.

It is probably true that any Anosov action has a periodic point. See [KLZ] for a special case.

We remark that if an orientable weakly Cartan action $\rho_{0}$ of $\Gamma$ on nilmanifold $M=N / \Lambda$ by automorphisms contains a "sufficiently large" locally rigid subaction (or several "sufficiently large" locally rigid subactions) of $\Gamma_{0} \subset \Gamma$, then $\rho_{0}$ is locally rigid. To be more specific, we recall that a small perturbation $\rho$ of the orientable weakly Cartan action $\rho_{0}$ is again orientable weakly Cartan action, and hence tangentially flat. Let the linearizing framing for $\rho$ be $\left\{X_{1}, \ldots, X_{n}\right\}$. Let $\Gamma_{1}, \ldots, \Gamma_{k} \subset \Gamma$ be subgroups so that for each $i=1, \ldots, k$ there exist $F_{i} \in \operatorname{Diff}^{r}(M)(r \geq 1)$,

$$
\rho\left(\gamma_{i}\right)=F_{i}^{-1} \circ \rho_{0}\left(\gamma_{i}\right) \circ F_{i} .
$$

We say that $\Gamma_{1}, \ldots, \Gamma_{k} \subset \Gamma$ fit $C^{r}$-tightly in an action $\rho_{0}$ of $\Gamma$ on nilmanifold $M=N / \Lambda$ by automorphisms if for any $C^{1}$-small smooth perturbation $\rho$ of $\rho_{0}$, $X_{1}, \ldots, X_{n}$ a linearizing framing for $\rho$, any $i=1, \ldots, n$, there exists $j_{i}$ such that $\left(F_{j_{i}}\right)_{*} X_{i}$ lifts to a right invariant vector field on $N$, where $F_{j_{i}} \in \operatorname{Diff}^{r}(M)$ satisfies (4.1), and $C^{0}$-close to $I d_{M}$.

It is easy to see that orientable Cartan actions of higher rank lattices on nilmanifolds contain tightly-fit abelian group actions. For more tightly-fit Anosov actions of higher rank lattices, we refer the reader to [Q2]. We state a corollary of Theorem 1.2 only for $C^{\infty}$-tightly-fit actions.

Corollary 4.3. Let $\rho_{0}$ be a $C^{\infty}$-orientable weakly Cartan action of $\Gamma$ on a nilmanifold $M$ by automorphisms containing $C^{\infty}$-tightly-fit subactions of $\Gamma_{1}, \ldots, \Gamma_{k} \subset \Gamma$. Then there exists a subgroup $\Gamma_{0} \in \Gamma$ such that $\rho$ is $C^{\infty}$-locally rigid; i.e., if $\rho$ is $C^{\infty}$ and $C^{1}$-close to $\rho_{0}$, there exists $F \in$ Diffo $(M) C^{0}$-close to Id, such that $\rho(\gamma)=F \rho_{0}(\gamma) F^{-1}$ for all $\gamma \in \Gamma_{0}$.

Proof. For any $C^{1}$-small perturbation $\rho$ of the orientable weakly Cartan action $\rho_{0}, \rho$ is again orientable weakly Cartan action, and hence tangentially flat for a subgroup $\Gamma_{0} \subset \Gamma$ of finite index (at most $2^{n}$ ). Let the linearizing framing for $\rho$ be $\sigma=\left\{X_{1}, \ldots, X_{n}\right\}$. Since there exist $C^{r}$-tightly-fit actions, $\sigma$ is smooth. Therefore, there exist $F \in \operatorname{Diff}^{\infty}(M)$ such that $\left.F \circ \rho\right|_{\Gamma_{0}} \circ F^{-1} \in \operatorname{Aff}(M)$. By the proof of Theorems 1.1 and 1.2, we may assume that $F$ is $C^{0}$-close to $I d_{M}$. By Stowe's stability of fixed point [S], we may also assume that there is a common fixed point for $\rho$ and $\rho_{0}$. So $\left.F \circ \rho\right|_{\Gamma_{0}} \circ F^{-1} \in \operatorname{Aut}(M)$. We note that $\operatorname{Aut}(M)$ is discrete, and we may choose $\rho C^{1}$-close enough to $\rho_{0}$. Therefore, that $F$ is $C^{0}$-close to $I d_{M}$ forces $\left.F \circ \rho\right|_{\Gamma_{0}} \circ F^{-1}=\left.\rho_{0}\right|_{\Gamma_{0}}$.

We remark that if $\rho_{0}$ is a Cartan action on an infra-nilmanifold $M$ with a fixed point, $\rho_{0}$ is locally rigid if $\rho(\Gamma)$ is generated by Anosov elements. Indeed, using 
an argument as in Corollary 4.3, we see that for any $C^{1}$-close action $\rho,\left.\rho\right|_{\Gamma_{0}}$ is conjugate to $\left.\rho_{0}\right|_{\Gamma_{0}}$. If $\rho\left(\Gamma_{0}\right)$ is generated by Anosov elements, we apply the following result essentially due to Palis and Yoccoz $[\mathrm{PY}]$ : If $A \in \operatorname{Aut}(M)$ is a hyperbolic automorphism and $f \in \operatorname{Homeo}(M)$ is a homeomorphism such that $A f=f A$, then $f \in \operatorname{Aut}(M)$. (For $M$ being a torus, see Proposition 0 of $[\mathrm{PY}]$, also see Proposition 2.1 of [KL1]. A different form of this assertion is appeared in Proposition 4.1.1 of [Q1]. For infra-nilmanifold, see the comment in the proof of Proposition 2.18 of [Hu1].) If $\gamma$ is an Anosov generator for $\rho_{0}$ and $\rho$ is $C^{1}$-close to $\rho_{0}, \gamma$ is also Anosov for $\rho$. Observing that there exists $k \geq 1$ such that $\rho\left(\gamma^{k}\right) \in \operatorname{Aut}(M)$ and $\rho(\gamma) \rho\left(\gamma^{k}\right)=\rho\left(\gamma^{k}\right) \rho(\gamma)$, we conclude that $\rho(\gamma) \in \operatorname{Aut}(M)$. We use the argument as in Corollary 4.3 again, and conclude that $\rho=\rho_{0}$ provided that $\rho$ is $C^{1}$-close enough to $\rho_{0}$.

\section{Proof of Lemma 3.3 FOR $r=1$}

We follow an argument of Weyl ([W], pp. 38-43) with a slight modification. We are grateful to G. D. Mostow for pointing this out and explaining to us the argument in $[\mathrm{M}]$.

We start with a result regarding the existence and uniqueness of a system of total differential equations. Let $x=\left(x^{1}, \ldots, x^{n}\right) \in \mathbb{R}^{n}, s=\left(s^{1}, \ldots, s^{r}\right) \in \mathbb{R}^{r}$, $F=\left(f_{i j}(s ; x)\right)$ be a $C^{1} n \times r$ matrix on an open set $E \subset \mathbb{R}^{n+r}$. Consider the system of total differential equations

$$
\frac{\partial x^{i}}{\partial s^{j}}=f_{i j}(s ; x) \text { for } i=1, \ldots, n ; j=1, \ldots, r
$$

with initial condition

$$
x\left(s_{0}\right)=x_{0}
$$

for some $\left(s_{0}, x_{0}\right) \in E$. If a $C^{1}$ solution $x=x(s)$ of (5.1) exists, $x(s)$ is of class $C^{2}$ ( since the right-hand side of (5.1) is of class $C^{1}$ ). Then $\partial^{2} x^{i} / \partial s^{j} \partial s^{k}=\partial^{2} x^{i} / \partial s^{k} \partial s^{j}$ leads to the condition

$$
\frac{\partial f_{i j}}{\partial s^{m}}+\sum_{k=1}^{n} \frac{\partial f_{i j}}{\partial x^{k}} f_{k m}=\frac{\partial f_{i m}}{\partial s^{j}}+\sum_{k=1}^{n} \frac{\partial f_{i m}}{\partial x^{k}} f_{k j}
$$

for $i=1, \ldots, n$ and $j, m=1, \ldots, r$ which must hold along the solution $(x, s)=$ $(x(s), s)$. Therefore, (5.3) is a necessary condition that (5.1) has a solution.

It turns out that (5.3) is also a sufficient condition for the existence and uniqueness of the solution of (5.1). For simplicity, we let $s_{0}=0$. Let

$$
J_{j m}^{i}(s ; x)=\left(\frac{\partial f_{i j}}{\partial s^{m}}-\frac{\partial f_{i m}}{\partial s^{j}}\right)+\sum_{k=1}^{n}\left(\frac{\partial f_{i j}}{\partial x^{k}} f_{k m}-\frac{\partial f_{i m}}{\partial x^{k}} f_{k j}\right) .
$$

We rewrite $(5.3)$ as

$$
J_{j m}^{i}(s ; x)=0 \quad \text { for all } j, m=1, \ldots, r .
$$

Proposition 5.1. Let $F=\left(f_{i j}(s ; x)\right)$ be a $C^{1} n \times r$ matrix on an open set $E \subset$ $\mathbb{R}^{n+r}$. Then (5.1) with initial condition $x(0)=x_{0}$ for $\left(0, x_{0}\right) \in E$ has a unique solution iff (5.4) is satisfied.

Proof. It is a direct corollary of Theorem VI.6.1, p 128 of [Har]. 
Proof of Lemma 3.3 for $r=1$. Let $M$ be smooth manifold of dimension $n,\left\{X_{i}\right\}_{i=1}^{n}$ be $C^{1}$ framings on $M,\left[X_{i}, X_{j}\right]=\sum_{k=1}^{n} C_{i j}^{k} X_{k}$. Let $m \in M$. By choosing a coordinate system about $m \in M$, we may assume that $m=0$ and there is a neighborhood $0 \in U_{0} \subset M$ so that $U_{0} \subset \mathbb{R}^{n}$. For each $j$, we write $X_{j}=\left(X_{1 j}, \ldots, X_{n j}\right)$. We let $F=\left(X_{i j}(x)\right)$, which is an $n \times n$ matrix of class $C^{1}$.

For any $C^{1} n \times n$ matrix $H=\left(h_{i j}\right)(s ; x)$ on an open set $0 \in E \subset \mathbb{R}^{2 n}$, we define $Z_{H}(s)=Z_{H}\left(s^{1}, \ldots, s^{n}\right)$ as follows. First we define $Z_{H}\left(s^{1}, 0, \ldots, 0\right)$ to be the solution of the initial value problem (of the system of ordinary differential equations)

$$
\left\{\begin{array}{l}
\frac{\partial x^{i}}{\partial s^{1}}=h_{i 1}\left(s^{1}, 0, \ldots, 0 ; x\right), \\
x^{i}(0)=0,
\end{array} \quad i=1, \ldots, n .\right.
$$

Next for fixed $s^{1}$, we define $Z_{H}\left(s^{1}, s^{2}, 0, \ldots, 0\right)$ to be the solution of the initial value problem (of the system of ordinary differential equations)

$$
\left\{\begin{array}{l}
\frac{\partial x^{i}}{\partial s^{2}}=h_{i 2}\left(s^{1}, s^{2}, 0, \ldots, 0 ; x\right), \\
x^{i}(0)=Z_{H}\left(s^{1}, 0, \ldots, 0\right),
\end{array} \quad i=1, \ldots, n .\right.
$$

Then we continue in this way step by step to define $Z_{H}(s)=Z_{H}\left(s^{1}, \ldots, s^{n}\right)$. We say that the function $Z_{H}(s)$ is associated with $H(s ; x)$ and initial condition $x(0)=0$. We remark that if $H$ is of class $C^{\gamma}, X_{H}(s)$ is a priori only of class $C^{\gamma}$.

Let $x(s)=Z_{F}(s)$ be associated with $F$ and the initial condition $x(0)=0$. Then $x(s)$ is of class $C^{1}$, and

$$
\frac{\partial x^{i}}{\partial s^{j}}\left(s^{1}, \ldots, s^{j}, 0, \ldots, 0\right)=X_{i j}\left(x\left(s^{1}, \ldots, s^{j}, 0, \ldots, 0\right)\right)
$$

by the construction of $Z_{F}(s)$. Let the continuous functions $\lambda_{i j}(s)$ be such that

$$
\frac{\partial x^{i}}{\partial s^{j}}=\sum_{k=1}^{n} \lambda_{k j} X_{i k}, \quad i, j, k=1, \ldots, n .
$$

It is easy to see that

$$
\lambda_{i j}\left(s^{1}, \ldots, s^{j}, 0 \ldots, 0\right)=\delta_{i j} .
$$

We shall show that $\lambda_{i j}(s)$ are analytic, and they only depend on the structure constants of $X_{1}, \ldots, X_{n}$ and condition (5.6).

We choose $X_{i}^{(l)}$ to be $C^{2}$ vector fields on $U_{0}$ so that $X_{i}^{(l)} \rightarrow X_{i}($ as $l \rightarrow \infty)$ in $C^{1}$ topology. Let $x_{l}(s)=Z_{H^{(l)}}(s)$ be associated with $H^{(l)}=\left(X_{i j}^{(l)}\right)$ with initial condition $x_{l}(0)=0$. We let the $C^{1}$-functions $\lambda_{i j}^{(l)}(s)$ be such that

$$
\frac{\partial x_{l}^{i}}{\partial s^{j}}=\sum_{k=1}^{n} \lambda_{k j}^{(l)} X_{i k}^{(l)}, \quad i, j, k=1, \ldots, n .
$$

Then

$$
\lambda_{i j}^{(l)}\left(s^{1}, \ldots, s^{j}, 0 \ldots, 0\right)=\delta_{i j}
$$

and

$$
\lambda_{i j}^{(l)}(s) \rightarrow \lambda_{i j}(s), \quad \text { as } l \rightarrow \infty
$$


in $C^{0}$-topology. Since (5.7) is solvable iff (5.4) is satisfied by Proposition 5.1, we obtain the following condition for $\lambda_{i j}^{(l)}(s)$ :

$$
\left(J^{(l)}\right)_{j m}^{i}:=\sum_{k=1}^{n}\left(\frac{\partial \lambda_{k j}^{(l)}}{\partial s^{m}}-\frac{\partial \lambda_{k m}^{(l)}}{\partial s^{j}}\right) X_{i k}^{(l)}+\sum_{p, q=1}^{n} \lambda_{p j}^{(l)} \lambda_{q m}^{(l)}\left[X_{q}^{(l)}, X_{p}^{(l)}\right]_{i}=0 .
$$

Let $\left[X_{p}^{(l)}, X_{q}^{(l)}\right]=\sum_{p, q, r}\left(C^{(l)}\right)_{p q}^{r}(x) X_{r}^{(l)}$. Then $\left(C^{(l)}\right)_{p q}^{r}(x) \rightarrow C_{p q}^{r}($ as $l \rightarrow \infty)$ in $C^{0}$-topology. Now (5.10) reduces to

$$
\left(\frac{\partial \lambda_{k j}^{(l)}}{\partial s^{m}}-\frac{\partial \lambda_{k m}^{(l)}}{\partial s^{j}}\right)+\sum_{p, q=1}^{n}\left(C^{(l)}\right)_{p q}^{k}(x) \lambda_{p j}^{(l)} \lambda_{q m}^{(l)}=0
$$

since $X_{1}, \ldots, X_{n}$ are independent. We now show that the limit of $\lambda_{k j}^{(l)}$ when $l \rightarrow \infty$ are analytic step by step by induction. Indeed with the help of (5.8), if $j<m$ and $s=\left(s^{1}, \ldots, s^{m-1}, 0, \ldots, 0\right),(5.11)$ reduces to

$$
\frac{\partial \lambda_{k j}^{(l)}}{\partial s^{m}}=\sum_{p}\left(C^{(l)}\right)_{p m}^{k} \lambda_{p j}^{(l)}
$$

Therefore, if we consider $s^{1}, \ldots, s^{m-1}$ as fixed, $\lambda_{k j}^{(l)}\left(s^{1}, \ldots, s^{m}, 0, \ldots, 0\right)$ is the solution of the system of ordinary differential equations (5.12) with initial condition $\lambda_{k j}^{(l)}\left(s^{1}, \ldots, s^{m-1}, 0, \ldots, 0\right)$. By induction hypothesis, $\lambda_{k j}^{(l)}\left(s^{1}, \ldots, s^{m-1}, 0, \ldots, 0\right) \rightarrow$ $\mu_{k j}\left(s^{1}, \ldots, s^{m-1}, 0, \ldots, 0\right)($ as $l \rightarrow \infty)$ in $C^{0}$-topology, which is analytic. By the continuous dependence of the solution with right-hand side functions and initial conditions, we see that the limit of $\lambda_{k j}^{(l)}\left(s^{1}, \ldots, s^{m}, 0, \ldots, 0\right)($ as $l \rightarrow \infty)$ is the solution of

$$
\frac{\partial \mu_{k j}}{\partial s^{m}}=\sum_{p} C_{p m}^{k} \mu_{p j}
$$

with initial condition $\mu_{k j}(0)=\mu_{k j}\left(s^{1}, \ldots, s^{m-1}, 0, \ldots, 0\right)$, hence analytic. Therefore, the limit of $\lambda_{k j}^{(l)}\left(s^{1}, \ldots, s^{n}\right)$ (as $l \rightarrow \infty$ ) is analytic by induction.

Combining this result and (5.9), we see that $\lambda_{k j}(s)$ are analytic, and only depending on the structure constants of $\left\{X_{1}, \ldots, X_{n}\right\}$. Moreover, $x(s)=X_{H}(s)$ is $C^{2}$.

Let $N$ be a smooth manifold of dimension $n,\left\{Y_{i}\right\}_{i=1}^{n}$ be $C^{1}$ framings on $N$, $\left[Y_{i}, Y_{j}\right]=\sum_{k=1}^{n} C_{i j}^{k} Y_{k}$. Let $n \in N$. By choosing a coordinate system about $n \in N$, we may assume that $n=0$ and there is a neighborhood $0 \in V_{0} \subset N$ so that $V_{0} \subset \mathbb{R}^{n}$. For each $j$, we write $Y_{j}=\left(Y_{j 1}, \ldots, Y_{j n}\right)$. We let $H=\left(Y_{i j}(y)\right)$, which is a $n \times n$ matrix of class $C^{1}$.

Let $y(s)=Y_{H}(s)$ be associated with $H$ and the initial condition $y(0)=0$. Similar argument yields that

$$
\frac{\partial y^{i}}{\partial s^{j}}=\sum_{k=1}^{n} \lambda_{k j}(s) Y_{i k}, \quad i, j, k=1, \ldots, n,
$$

for the same analytic $\lambda_{k j}$ as in (5.7).

Now after possibly shrinking the neighborhoods $m \in U_{0}, n \in V_{0}$ (say $m \in U \subset$ $\left.U_{0}, n \in V \subset V_{0}\right)$, the diffeomorphism $F_{*}$ from $U \rightarrow V$ by composing $y=y(s)$ with the inverse of $x=x(s)$ is clearly the desired diffeomorphism. 
We remark that the approximation argument (in our case $r=1$ ) is needed. Since the right-hand side of

$$
\frac{\partial x^{i}}{\partial s^{j}}=\sum_{k=1}^{n} \lambda_{k j} X_{i k}, \quad i, j, k=1, \ldots, n,
$$

is a priori only continuous, we cannot use Proposition 5.1 directly. Our argument of course applies to all cases $r \geq 1$. For $r \geq 2$, we can use Proposition 5.1 directly to (5.14), which is the argument of Weyl in [W].

\section{REFERENCES}

[A] D. V. Anosov, Geodesic flows on closed Riemannian manifolds with negative curvature, (English translation, A.M.S., Providence, RI, 1969), Proceedings of the Steklov Institute of Mathematics 90 (1967), 1-235. MR 36:7157

[BP] M.I. Brin and Ya.B. Pesin, Partially hyperbolic dynamical systems, Math USSR Izvestija 8 (1) (1974), 177-218. MR 49:8058

[F] R. Feres, Actions of discrete linear groups and Zimmer's conjecture, J. Differential Geom. 42 (3) (1995), 554-576. CMP 96:06

[Har] P. Hartman, Ordinary differential equations, John Wiley and Sons, Inc., 1973. MR 49:9294

[HP] M.W. Hirsch and C.C. Pugh, Stable manifolds and hyperbolic sets, in Global Analysis, Vol XIV, Proceedings of Symposia in Pure Mathematics, AMS, Providence, R.I., 1970. MR 42:6872

[Hu1] S. Hurder, Rigidity for Anosov actions of higher rank lattices, Ann. of Math. 135 (1992), 361-410. MR 93c:22018

[Hu2] - Topological rigidity of strong stable foliations for Cartan actions, Erg. Th. \& Dyn. Sys. 14 (1994), 151-167. MR 95f:58060

[KL1] A. Katok and J. Lewis, Local rigidity for certain groups of toral automorphisms, Israel Math. Jour. 75 (1991), 203-241. MR 93g:58076

[KL2] - Global rigidity results for lattice actions on tori and new examples of volumepreserving actions, Israel J. Math. 93 (1996), 253-280. CMP 96:10

[KLZ] A. Katok, J. Lewis and R. Zimmer, Cocycle superrigidity and Rigidity for lattice actions on tori, Topology 35 (1) (1996), 27-38. CMP 96:06

[L] J. Lewis, The algebraic hull of the derivative cocycle, Preprint (1993).

[Li] A. Livsic, Cohomology of dynamical systems, Math. USSR-Izv. 6 (1972), 1278-1301. MR 48: 12606

[M] G. D. Mostow, personal communication, 1994.

[PY] J. Palis and J.C. Yoccoz, Centralizers of Anosov diffeomorphisms on tori, Ann. Scient. Èc.Norm. Sup. 22 (1989), 99-108. MR 90i:58151b

[PR] G. Prasad and M. S. Raghunathan, Cartan subgroups and lattices in semisimple groups, Ann. Math. 96 (1972), 296-317. MR 46:1965

[Q1] N. Qian, Anosov automorphisms for nilmanifolds and rigidity of group actions, Ergodic Theory Dynam. Systems 15 (2) (1995), 341-359. MR 96g:58147

[Q2] - Smooth conjugacy for Anosov diffeomorphisms and rigidity of Anosov actions of higher rank lattices, Preprint.

[QZ] N. Qian and R.J. Zimmer, Entropy rigidity for semisimple group actions, to appear Israel J. Math.

[S] D. Stowe, The stationary set of a group action, Proc. AMS 79 (1980), 139-146. MR 81b:57035

[T] W. Thurston, The geometry and topology of 3-manifolds, Lecture notes, Princeton.

[W] H. Weyl, The structure and representation of continuous groups, Institute for Advanced Studies, 1934

[Z1] R. Zimmer, Orbit equivalence and rigidity of ergodic actions of Lie groups, Erg. Th. \& Dyn. Sys. 1 (1981), 237-253. MR 84a:22019

[Z2] _ Ergodic theory and semisimple groups, Birkhäuser, Boston, 1984. MR 86j:22014

[Z3] - Actions of semisimple groups and discrete subgroups, Proceedings of the International Congress of Mathematicians, Berkeley (1986), 1247-1258. MR 89j:22024 
[Z4] - On the algebraic hull of an automorphism group of a principal bundle, Comment. Math. Helvetici 65 (1990), 375-387. MR 92f:57050

[Z5] _ Actions of simple Lie groups, in Workshop on Lie groups, ergodic theory and geometry and problems in geometric rigidity, 1992.

Department of Mathematics, Yale University, P.O. Box 208283, New Haven, ConNECTICUT 06520

E-mail address: qian@math.yale.edu 